\title{
A Survey of Knowledge and Beliefs about Antimicrobials use among Prescribers in a Teaching Tertiary Care Hospital
}

\author{
Authors \\ Dr Shivani Juneja, Dr Shalini Chawla, Anmol Kanwar, Anushka Aggarwal \\ Maulana Azad Medical College
}

\section{Introduction}

Misconceptions and lack of basic knowledge about the use of antimicrobial agents (AMAs) use seems to be an important aspect in their prescribing in both developed and developing countries. ${ }^{[1]}$ The lack of appropriate knowledge in this direction has contributed to the development of antimicrobial resistance. This has become a worldwide public health problem leading to serious hospital and community acquired infections. Moreover, the emergence of multidrug resistant bacteria are casting a negative impact on treatment outcomes such as prolonged morbidity, hospital stay and increased risk of mortality. ${ }^{[2,3]}$

A successful management of antimicrobial use requires robust information on the use of antimicrobials. This will enable a better understanding of the evolving relationship between antimicrobial consumption and the emergence of resistance. ${ }^{[3]}$ According to a study, a better understanding of what physicians know about issues of AMAs use and resistance can enhance the effectiveness of interventions targeted at improving in-hospital antimicrobial use. ${ }^{[4,5]}$

The present study is a preliminary step to know about the prevailing knowledge regarding antimicrobial use and the beliefs associated with them in Maulana Azad
Medical College (MAMC) and associated hospitals. This study was planned with an objective to utilize the information obtained from this survey, in planning the intervention to educate the prescribers regarding the use of AMAs.

\section{Aims and Objective}

To evaluate the knowledge and beliefs about antimicrobial prescribing among prescribers in a teaching tertiary care hospital

\section{Material and Methods}

This study was carried out in the Department of Pharmacology, MAMC and associated hospitals, New Delhi. The study was conducted after obtaining clearance from the Institutional Ethics Committee.

Study Design: This was a prospective, crosssectional study which was carried out in the form of a survey. In this study a pretested, semi-structured, validated questionnaire was administered to the prescribers of different specialties of the hospital. Three hundred thirty (330) prescribers were surveyed; the data was collected and analyzed using the descriptive statistics. 


\section{Results}

A total of 330 prescribers filled in the questionnaire. This comprised of 50 Consultants (Cons), 50 Senior Residents (SRs), 130 Junior Residents (JRs) and 90Interns (Int) of various departments of the hospital. The questionnaire consisted of 18 questions based upon the prescription of AMAs in the patients. (Table 1)

Out of 330 subjects, $156(47.27 \%)$ believed that all bacterial, fungal and viral infections (62\% Cons, $58 \%$ SRs, 53\% JRs, 50\% Interns); and 124 (37.57\%) believed that both bacterial and fungal infections can be treated with AMAs.

When asked about the speeding up of recovery from illness by AMAs,

221(66.96\%) prescribers agreed that AMAs speed up recovery. Seventy three $(73 \%)$ percent respondents believed that branded and more expensive AMAs did not have a better efficacy. Regarding the prevention of infections with AMAs, $42 \%$ prescribers believe AMAs can be prescribed for the prevention of various infections.

Two hundred fifty eight out of 330 prescribers (78\%) do not prescribe AMAs to all the patients who present with cough, cold and fever. Out of $78 \%$ prescribers, $86 \%$ were Cons, $76 \%$ SRs, $79 \%$ JRs and $69 \%$ were Interns. Eighty nine (89\%) percent of the respondents do not prescribe AMAs from the same class concomitantly.

In response to the most common reason for prescribing AMAs, pyrexia was found to be the most common one (Cons $48 \%$, SRs $30 \%$, JRs $42 \%$, Interns $44 \%$ ) followed by diarrhea, common cold and respiratory infections.Figure1 shows the percentage response with regard to a question; if the same AMA will always be effective in the treatment of similar infection in the future for the same patient. Another aspect was the empirical use of AMAs. In this context $82 \%$ Cons, $84 \%$ SRs, $82 \%$ JRs and $73 \%$ Interns were aware about the use of AMAs empirically. Out of them 58\% Cons, 50\% SRs, 67\% JRs and 63\% Interns tried to justify the empirical use of AMAs. Even though they were asked to specify the reason but most of them did not mention the same.

In addition to this, in reply to the question that what all information is required before initiation of antimicrobial therapy; the most common responses were history of allergy, laboratory data for culture reports as shown in Figure 2.Fifty six percent consultants, 50\% SRs, 51\% JRs and 65\% Interns believe that the ongoing AMA therapy should be reviewed after 72 hours of initiation as shown in Table 2.The points being taken care of while reviewing antimicrobials as asked in question 13 (Table1) included all of them according to Center for Disease Control (CDC). In response to this question, $88 \%$ Cons, 64\% SRs, 64\%JRs and 58\% Interns were in favour of all these points to be taken care of while reviewing AMAs.

As far as the knowledge of antibiogram along with its importance is concerned, it was observed that $62 \%$ Cons, 52\% SRs, 54\% JRs and 56\% Interns had some idea about the existence of antibiogram. And out of them $58 \%$ Cons, $46 \%$ SRs, $48 \%$ JRs and 54\% Interns knew that our hospital have an antibiogram.

Three hundred ten out of total 330 prescribers (94\%) think overuse and misuse of antimicrobials has bearing in developing antimicrobial resistance (Figure 3). The over the counter use of AMAs is rising due to increased antimicrobial prescription as believed by 96\% Cons, 96\% SRs, 93\% JRs and 99\% Interns. Ninety two percent of the prescribers think that antimicrobial prescribing need extra emphasis as a special category drugs at the undergraduate level. 


\section{JMSCR Vol||06||Issue||03||Page 642-647||March}

Figure1: Do you think same antimicrobial will always be effective in the treatment of similar infection in the future for the same patient?

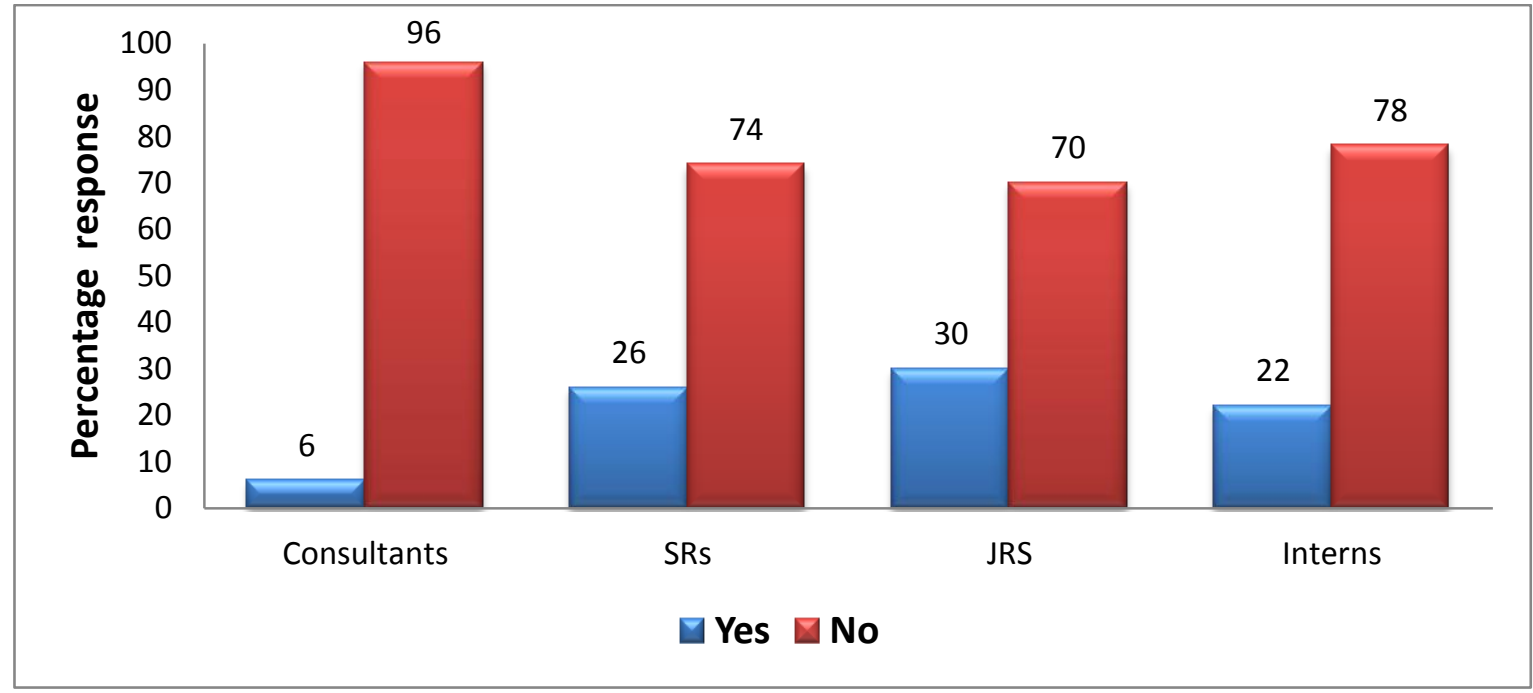

Figure 2: What all information is required before initiation of AMA therapy?

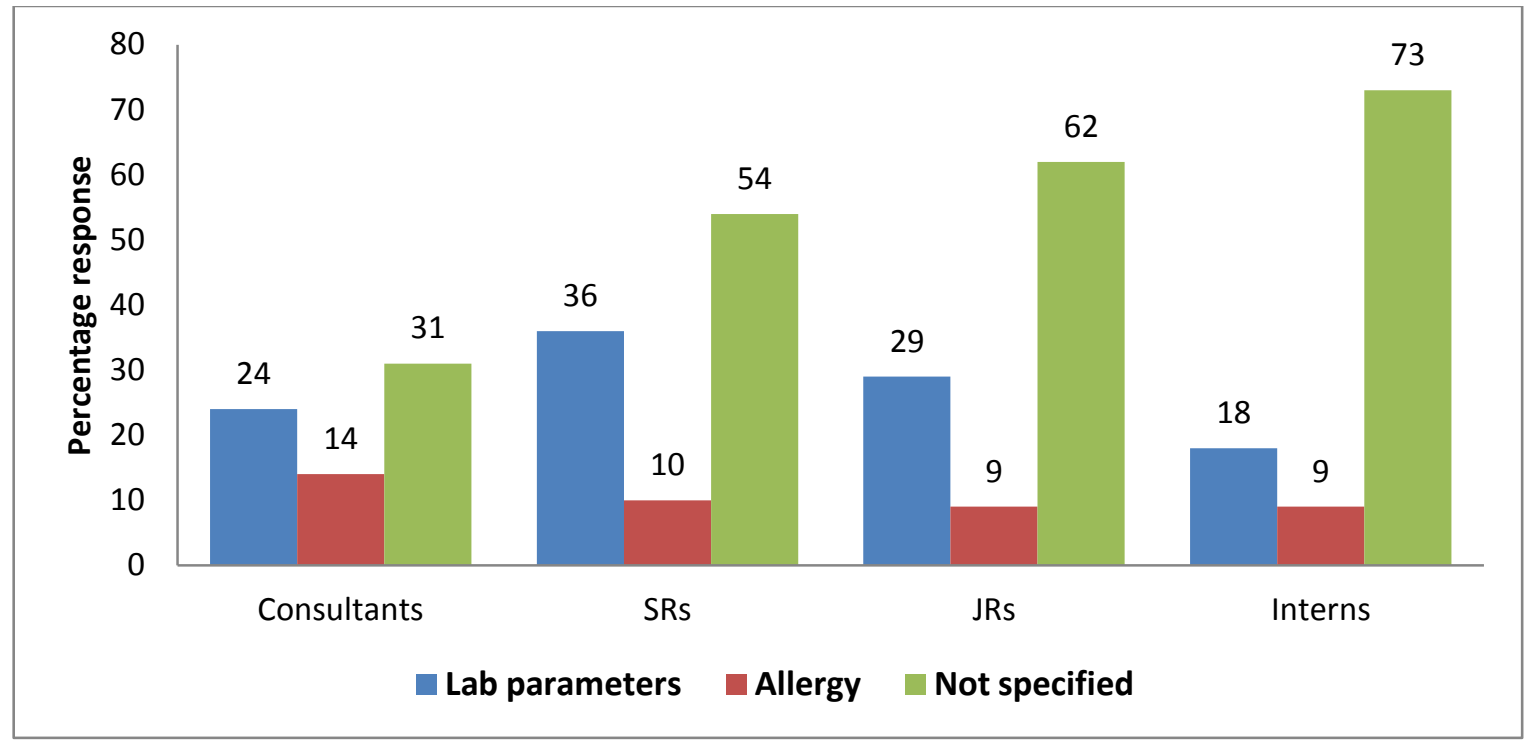

Figure 3: Do you think overuse and misuse of antimicrobials has any bearing in developing antimicrobial resistance?

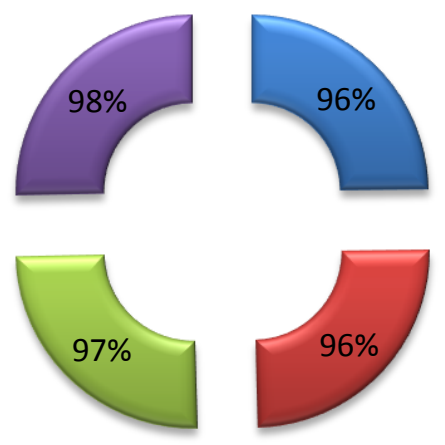

$\square$ Consultants $\square$ SRs $\square$ JRs $\square$ Interns 
Table 1: Questionnaire

A Survey of knowledge and beliefs of antimicrobial use among the prescribers of MAMC and associated hospitals

Designation: Intern/JR/SR/Consultant

(Kindly tick mark the right choice. There could be more than one right choice)

1. Which infections are treated with antimicrobials?
a. Bacterial
b. Viral
c. Fungal
d. All

2. Do antimicrobials speed up recovery from infections?

a. Yes
b. No

3. Do branded and more expensive antimicrobials have better efficacy?

$\begin{array}{ll}\text { a. } & \text { Yes } \\ \text { b. No }\end{array}$

4. Can antimicrobials be prescribed for prevention of infections?

a. Yes
b. No

5. Do you prescribe antimicrobials to all patients presenting with cough, cold and fever?
a. Yes
b. No

6. Do you prescribe antimicrobials from the same class concomitantly?
a. Yes
b. No
If yes, specify

7. According to you, what could be your most common reason for prescribing antimicrobials?
a. Common cold
b. Diarrhoea
c. Pyrexia
d. Others, specify

8. Do you think same antimicrobial will always be effective in the treatment of similar infection in the future for the same patient?
a. Yes
b. No

9. Do you know about empirical use of antimicrobials?
a. Yes
b. No
If yes, specify

10. If yes, do you justify empirical use of antimicrobials?
a. Yes
b. No
Specify

11. What all information is required before initiation of antimicrobial therapy? Specify

12. After how long will you review the ongoing antimicrobial therapy?
a. 24 hours
b. 48 hours
c. 72 hours
d. 5 days

13. Which of the following points are taken care of, by you, while reviewing antimicrobials?
a. Does this patient have an infection that will respond?
b. If yes, is the patient on right antimicrobial with right dose and route of administration?
c. Can a better targeted antibiotic be used to treat infection?
d. How long should the patient receive this therapy?

14. Do you have any idea about hospital antibiogram?
a. Yes
b. No

15. If yes, do you know if our hospitals have an antibiogram?
a. Yes
b. No

16. Do you think overuse and misuse of antimicrobials has any bearing in developing antimicrobial resistance?
a. Yes
b. No

17. Do you think increased antimicrobial prescription is promoting their over the counter use?

a. Yes

18. Do you think prescription of antimicrobials need extra emphasis as a special category drugs at the undergraduate level? $\begin{array}{ll}\text { a. } & \text { Yes } \\ \text { b. No }\end{array}$ 
Table 2: After how long will you review the ongoing antimicrobial therapy?

\begin{tabular}{|l|c|c|c|c|}
\hline Duration & Consultants (\%) & Senior Residents (\%) & Junior Residents (\%) & Interns (\%) \\
\hline $\mathbf{2 4}$ hours & 2 & 6 & 2 & 3 \\
\hline $\mathbf{4 8}$ hours & 24 & 24 & 18 & 12 \\
\hline $\mathbf{7 2}$ hours & 56 & 50 & 51 & 65 \\
\hline 5 days & 18 & 20 & 29 & 20 \\
\hline
\end{tabular}

\section{Discussion}

This study was done with an aim to acknowledge the problem of growing antimicrobial resistance due to irresponsible prescribing practicing. Majority of prescribers and mainly the consultants were not in favour of prescribing AMAs in self-limiting viral illnesses. ${ }^{[6]}$

Empirical treatment is when the therapy is started without knowing the exact cause/nature of the disorder based upon the experience. The broad spectrum antimicrobial is started till the bacterial culture or related test reports are awaited. As the prescribers were from different levels in this study, so as compared to consultants; interns when asked were in a difficult position to explain the empirical use of AMAs. ${ }^{[7]}$

Furthermore, as known that anantibiogram is a periodic summary of antimicrobial susceptibilities of local bacterial isolates of a particular hospital. Most of the prescriptions nowadays are made based upon them to combat unnecessary prescribing of AMAs. ${ }^{[8]}$ So the finding in this study suggests that most of the prescribers were unaware of the existing antibiograms and their utility in the prescription of AMAs.

At the various levels of the prescribers from consultants to Interns, it was observed that the knowledge of all levels was comparable for few questions that were asked about AMA prescription. But for some, it was found that prescribers at the level of consultants had a better knowledge especially in the context of reviewing of AMA therapy as well as the empirical use.

Our findings suggest $92 \%$ prescribers want some education to be emphasized in AMAs prescribing at the undergraduate level to address the issues related to AMA prescription. ${ }^{[9,10]}$ This finding is well in accordance with a study by Srinivisan et al where by $90 \%$ of house staff wanted more teaching about antimicrobials. ${ }^{[11]}$

\section{Conclusion}

This study is a preliminary step to emphasize the importance of antibiotic stewardship. It is required to combat the unnecessary use of AMAs at the level of prescribers; a mandate to prevent evolving AMA resistance in the long run. The available medical education may need some extra emphasis at the undergraduate level for a better perspective in this direction. Any action today in this direction shall only result in a better future.

\section{Conflict of Interest: None}

\section{References}

1. Knowledge and beliefs about antibiotics among people in Yogyakarta City Indonesia: a cross sectional population-based survey. Antimicrobial Resistance and Infection Control. 2012;1:38.

2. Abera et al.: Knowledge and beliefs on antimicrobial resistance among physicians and nurses in hospitals in Amhara Region, Ethiopia. BMC Pharmacology and Toxicology. 2014;15:26.

3. Widayati et al. Knowledge and beliefs about antibiotics among people in Yogyakarta City Indonesia: a cross sectional population-based survey Antimicrobial Resistance and Infection Control. 2012;1:38

4. Gyssens, I.C. Quality measures of antimicrobial drug use. Int. J. Antimicrob. Agents. 2001;17: 9-19.

5. Charani E, Cooke J, Holmes A. Antibiotic stewardship programmes - what's missing? J AntimicrobChemother 2010; 65:2275-7.

6. Garcia C, Llamocca LP, Garcia K, Jimenez A, Samalvides F, et al. Knowledge, attitudes and practice survey about antimicrobial resistance and prescribing among physicians in a 
hospital setting in Lima, Peru. BMC Clinical Pharmacology. 2011;11:18.

7. Mettler J, Simcock M, Sendi P,Widmer AF, Bingisser R,Battegay M, et al. Empirical use of antibiotics and adjustment of empirical antibiotic therapies in a university hospital: a prospective observational study. BMC Inf Dis. 2007;7:21.

8. S. Joshi. Hospital antibiogram: a necessity. Indian J Med Microbiol. 2010; 28: 277-280.

9. Huang Y, Gu J, Zhang M, Ren Z, Yang W, Chen $\mathrm{Y}$, et al.Knowledge, attitude and practice of antibiotics: a questionnaire study among 2500 Chinese students. BMC Medical Education. 2013;13:163.

10. Mahajan M, Dudhgaonkar S, Deshmukh S. A Questionnaire based Survey on the Knowledge, Attitude and Practises about Antimicrobial Resistance and Usage among the Second year MBBS Students of a Teaching tertiary care Hospital in Central India. IJPR. 2014;4:175-79.

11. Srinivasan A, Song X, Richards A, SinkowitzCochran R, Cardo D, Rand C.A Survey of Knowledge, Attitudes, and Beliefs of House Staff Physicians From Various Specialties Concerning Antimicrobial Use and Resistance. Arch Intern Med. 2004;164:145156. 